\title{
Abdominal Dermolipectomy at Laparotomy with Huge Ovarian Benign Tumor
}

\section{Ryotaro Nagao ${ }^{1}$, Hiroshi Takagi ${ }^{1}$, Chinatsu Koiwai ${ }^{1}$, Satoshi Ichigo ${ }^{1}$, Hiroyuki Kajikawa ${ }^{1}$, Kazutoshi} Matsunami ${ }^{1}$, Masato Shiba ${ }^{2}$, Takeshi Kitazawa ${ }^{2}$ and Atsushi Imai*1

${ }^{1}$ Departments of Obstetrics and Gynecology, Gifu Prefecture, Japan

${ }^{2}$ Plastic Surgery, Matsunami General Hospital, Gifu Prefecture, Japan

Received: January 15, 2018; Published: January 19, 2018

*Corresponding author: Atsushi Imai, M.D., Institute of Endocrine-Related Cancer, Matsunami General Hospital, Kasamatsu, Gifu 501-6062, Japan, Tel: 81 583880111; E-mail: aimai@matsunami-hsp.org.jp

\section{Abstract}

Most of obese patients have more comorbidity, longer operation times, longer hospital stays, and more early and late complications. This report presents a case of severe obesity with large amounts of redundant skin and fat extending to lower abdomen, in which a abdominal dermolipectomy was carried out at the time of laparotomy against giant ovarian mature cysticteratoma.

Keywords: Abdominoplasty; Dermolipectomy; Benign Ovarian Tumor; Laparotomy; Incisional Negative Pressure Wound Therapy

\section{Introduction}

Obesity is a common health problem and defined as a body mass index (BMI) of $30 \mathrm{~kg} / \mathrm{m}^{2}$ or greater; morbid obesity is defined as a BMI $40 \mathrm{~kg} / \mathrm{m}^{2}$ or greater. Apart from the limited securing an operative view, obesity has a significantly higher risk of postoperative deep vein thrombosis, surgical site infections, nerve injury, and urinary infection $[1,2]$. Abdominoplasty and dermolipectomy are originally a form of cosmetic surgery used to make the abdomen tighter and defined as a surgical procedure in which excess skin and fat in the abdominal area is removed $[3,4]$. We present one patient of severe obesity with large amounts of redundant skin and fat extending to lower abdomen, in whom an abdominal dermolipectomy was performed at the time of laparotomy against giant ovarian tumor. (Figure 1).

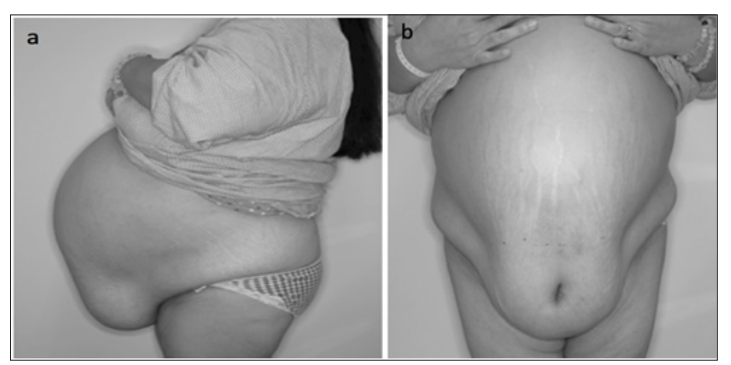

Figure 1: Preoperative anterior view (a) and lateral view (b). Excessive soft tissue in the abdomen forms an apronlike appearance.

\section{Case report}
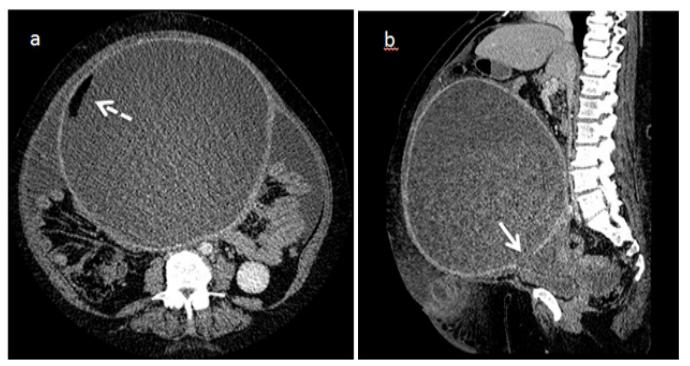

Figure 2: CT image of the patient pelvis. Enlarged cystic mass with fat component (dot arrow) and perforation of cyst wall (solid arrow) were demonstrated.

A 45-year-old woman, G3P1, was scheduled for exploratory laparotomy because of huge ovarian cystic tumor. She had 3-year history of progressive abdominal distention. The patient was morbidly obese, with a weight of $107 \mathrm{~kg}$ and height of $158 \mathrm{~cm}$ (bodymass index (BMI): 42.9). Physical examination revealed excessive soft tissue in the abdomen forming an apron-like deformity. Preoperative laboratory test showed a hemoglobin $6.4 \mathrm{~g} / \mathrm{dl}$ and Fe of $13 \mu \mathrm{g} / \mathrm{dl}$, and renal function and liver function were within normal. Serum levels of tumor markers were as follows: CEA, 25.6ng/ml (reference range < 5); CA19-9 $173.6 \mathrm{U} / \mathrm{ml}(<37$ ); CA125, $291 \mathrm{U} / \mathrm{ml}(<35)$. Oral glucose tolerance test and insulin release test were both normal. Preoperative chest roentgenogram 
and a transthoratic echocardiogram revealed normal. Ultrasound and CT examination showed $27 \times 25 \times 22 \mathrm{~cm}$ unilobular cystic mass with a smooth surface and fat-containing fluid with break in the wall continuity (Figure 2). Those finding, combined with the physical and laboratory findings, lead to a diagnosis probable ovarian dermoid cyst and its partial rupture.

The patient underwent an abdominoplasty and a lapatotomy. The operation began with a lower abdominal midline incision crossing the transverse incision on fold of the apron-like deformity in a "gull wing" fashion. The caudally located abdominal tissue flap was elevated from the fascia and then followed with laparotomy including bilateral salpingo-oophorectomy. The abdominal flap was then pulled downwards and excess abdominal wall tissue was excised (Figure 3). Closure was performed with isolated Vicryl 3.0 sutures and running intracutaneous monocryl 3.0 sutures (Figure 3). Incisional negative pressure wound therapy was applied to the wound. A histological diagnosis was benign denatured mature cystic teratoma originated from right ovary. Postoperative symptoms improved over 1 week, and the patient was discharged on the 10th postoperative day.

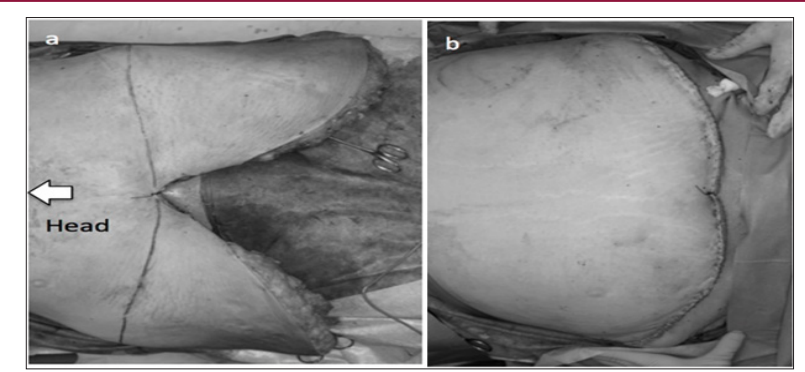

Figure 3: Incision marking before excess abdominal wall excision (a) and final result (b). Umbilicus was dissected because of continuity to ovarian cyst.

\section{Comments}

Most of obese patients have a pendulous abdomen and bad odor caused by fungal infection in the skin folds [3]. They have more comorbidity, longer operation times, longer hospital stays, more early and late complications than other group of patients [1]. To our knowledge, no recent study in the literature has been evaluated advantages for abdominoplasty and dermolipectomy at laparotomy in benign gynecologic practice. [5]. Demonstrated that this procedure provided two important advantages for stoma surgery, stability and better postoperative care by the patient. At the time of knee surgery, a large area of the patient's lower abdomen may become ischemic, thus making abdominoplasty the only option for treatment [6]. In our patient in whom a laparotomy was performed through a midline incision, we also carried out an abdominoplasty at reducing abdominal wall excess. This concurrent surgical procedure may be very attractive as a safe and minimally invasive technique in relation to the postoperative management for obese patients.

\section{References}

1. Ersoy E, Evliyaoğlu Ö, Erol O, Ersoy A, Akgül M, et al. (2016) Effects of the morbid obesity and skin incision choices on surgical outcomes in patients undergoing total abdominal hysterectomy. Turk J Obstet Gynecol 13: 189-195.

2. Blikkendaal M, Schepers E, van Zwet E, Twijnstra A, et al. (2015) A systematic review with cumulative analysis of comparative studies. Arch Gynecol Obstet 292: 723-738.

3. Murshid M, Khalid K, Shakir A, Bener A (2010) Abdominoplasty in obese and in morbidly obese patients. Plast Reconstr Aesthet Surg 63: 820825.

4. Halbesma G, Van der Lei B (2008) The reverse abdominoplasty: A report of seven cases and a review of english-language literature. Ann Plast Surg 61: 133-137.

5. Yazawa M, Kotake K, Matsui T, Asaga S (2007) Abdominal dermolipectomy in laparotomy with stoma surgery: Case report. Surg Technol Int 16: 37 40.

6. Haritopoulos K, Labruzzo C, Papalois V, Hakim N (2002) Abdominoplasty in a patient with severe obesity. Int Surg 87: 15-18.

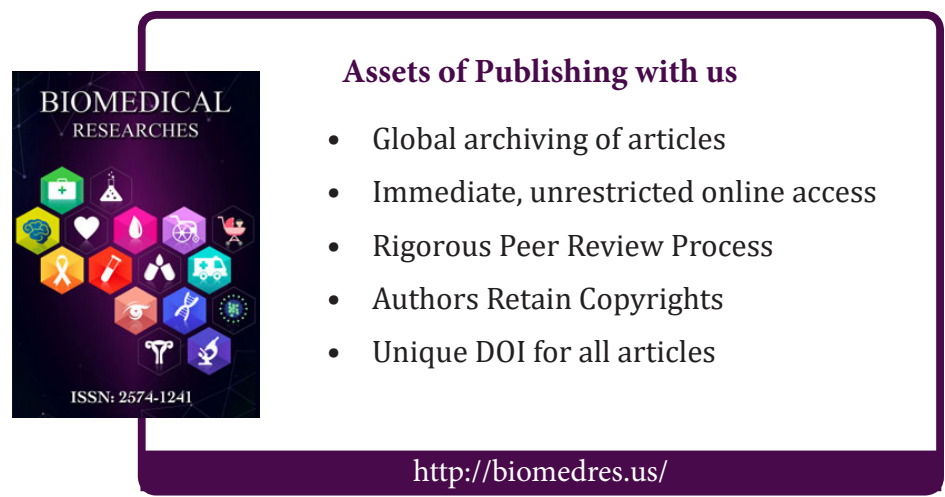

